\title{
Fingerprinting quantum emitters in hexagonal boron nitride using strain
}

\author{
Pratibha Dev (1) \\ Department of Physics and Astronomy, Howard University, Washington, DC 20059, USA
}

(Received 18 February 2020; accepted 21 May 2020; published 5 June 2020)

\begin{abstract}
Two-dimensional van der Waals crystals and their heterostructures provide an exciting alternative to bulk wide-band-gap semiconductors as hosts of single-photon emitters. Among different layered materials, bright and robust defect-based single-photon emitters have been observed within hexagonal boron nitride, a layered wide-band-gap semiconductor. Despite research efforts to date, the identities of the deep defects responsible for quantum emissions in hexagonal boron nitride remain unknown. In this theoretical work, I demonstrate that the strain-induced changes in emission frequencies depend on (i) the detailed nature of the defect states involved in the optical excitations, and (ii) the rich boron chemistry that results in complex interactions between boron atoms. As each defect shows a distinct response to the strain, it can be used not only to tune emission frequencies but also to identify the quantum emitters in hexagonal boron nitride.
\end{abstract}

DOI: 10.1103/PhysRevResearch.2.022050

\section{INTRODUCTION}

The discovery of quantum emitters in different twodimensional (2D) layered structures is a significant development in the search for qubit candidates for quantum technologies [1-12]. These van der Waals crystals and their heterostructures provide an exciting alternative to quantum emitters (QEs) within bulk wide-band-gap semiconductors. The surface-only structure of the host 2D materials allows for a facile manipulation of the electronic-structure properties of the 2D layers, and hence the QEs within them, via different means, such as changes in composition at the atomistic level $[7,13]$, creation of heterostructures of 2D layers [14-16], or through application of strain $[9,10]$.

To date, the nature of quantum emitters in layered materials has remained unclear. Even when it is determined that the quantum emitter is a deep defect, as in the case of hexagonal boron nitride (hBN) [5-9,11,12], the exact identity remains unknown. In the case of hBN, experimental attempts to identify the defects are confounded by the widely varying properties of the QEs, such as their brightness, emission frequencies, and polarizations [6,9,11,17-19]. These variations are observed not only in different samples, which may be prepared using very different treatments, but also within a sample. The emission frequencies of QEs in hBN range from ultraviolet to near infrared $[5,6,9,19,20]$. The emitters are often grouped according to their zero phonon line (ZPL) energies and phonon sideband shapes [6]. Different groups of emitters possibly correspond to chemically different defects, or defects with different charge states, or a combination of

\footnotetext{
*pratibha.dev@howard.edu
}

Published by the American Physical Society under the terms of the Creative Commons Attribution 4.0 International license. Further distribution of this work must maintain attribution to the author(s) and the published article's title, journal citation, and DOI. both. Even for what appears to be the same defect in a sample, a large spectral distribution in the ZPL frequencies is reported $[6,9]$. This distribution is usually attributed to different local strains around a QE species. Hence, understanding how strain affects properties of QEs is an important step towards fully characterizing them. Only a few experiments exist that have directly studied the effect of strain on ZPL frequencies of QEs in $\mathrm{hBN}[9,21]$. On the theoretical side, an understanding of the response to strain for different defects has been mostly missing.

In this work I address these issues by studying the effects of strain on the excited-state properties of different candidate defects within $\mathrm{hBN}$ and show how the response of the defects to the strain can be used to identify the defects themselves. Strain displaces the ions, affecting the orbital degrees of freedom of pointlike defects. Energies of the defect statesmolecular orbitals constructed from the dangling bonds at the defect site-are modified by the strain, tuning the excitation energies according to $H_{\text {strain }}=\sum_{\Gamma} \Lambda_{\Gamma} \epsilon_{\Gamma}$, where the strain Hamiltonian, $H_{\text {strain }}$, has been projected onto the irreducible representations $(\Gamma)$ of the defect's symmetry group, $\Lambda_{\Gamma}$ are the orbital operators, and $\epsilon_{\Gamma}$ are the strain tensors. In order to keep the study focused, I concentrate on the symmetrypreserving hydrostatic strain (dilation and contraction), which belongs to the most symmetric representation of the group and merely shifts the energies of the defect states. As the makeup of the defect states is unique for each defect, the strain-induced modification in defect properties varies from defect to defect. Another important consideration is that undercoordinated boron atoms are involved in the defects. The boron atom occupies a special position in the periodic table due to its highly unconventional chemistry, with a tendency to form multicenter bonds to overcome electronic frustration. I show that the rich chemistry of boron plays a role in the equilibrium geometries adopted by the defects in their ground and excited states. In turn, this influences the effects of strain on the ZPL. 
(a)

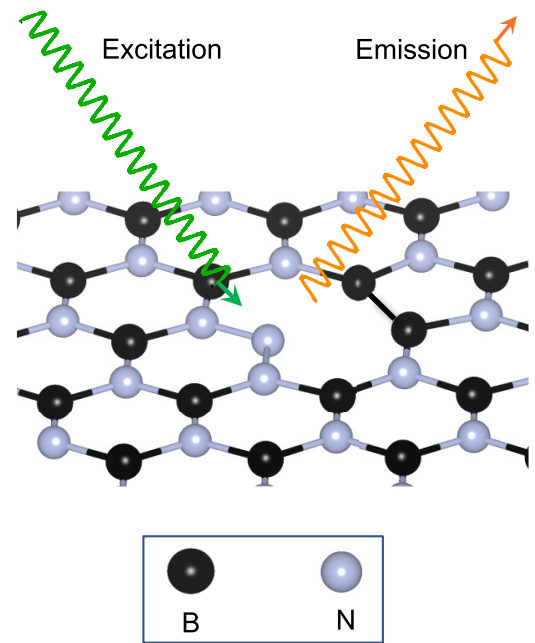

(b)

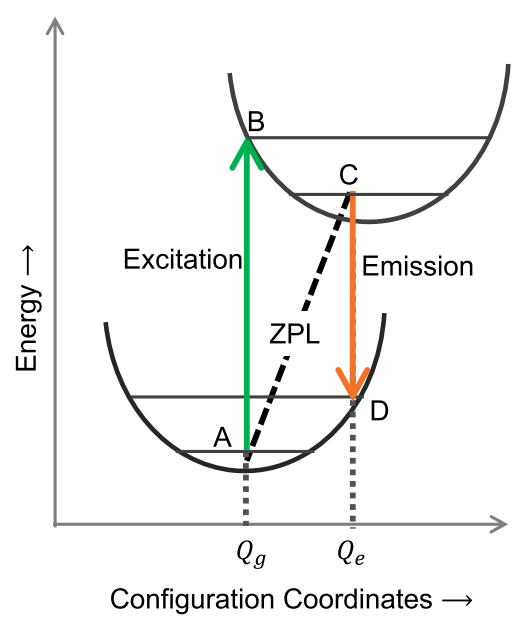

(c)

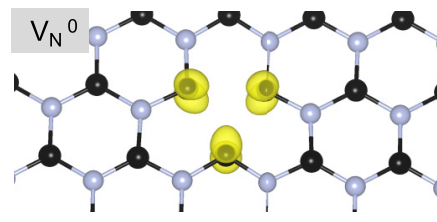

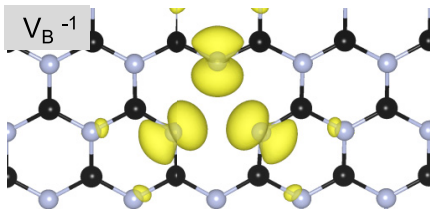

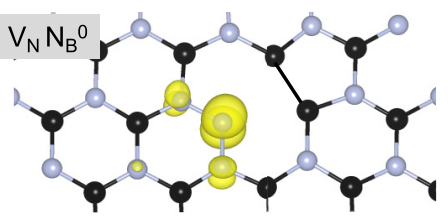

FIG. 1. Deep defects in hBN as quantum emitters. (a) Schematic of hBN lattice with a defect undergoing photoluminescence (PL) process. (b) Franck-Condon picture used to mimic PL within constrained-occupation DFT (CDFT). The configuration coordinate diagram is a plot of total energy as a function of ground (bottom) and excited-state (top) configurations. Within CDFT, total energies corresponding to points A, $\mathrm{B}, \mathrm{C}$, and D are calculated by promoting an electron from a filled defect state to a higher (formerly empty) state to mimic the PL process. (c) Spin-density plot [difference in charge densities within majority and minority-spin channels] for defects in unstrained hBN showing the localized nature of the defect states.

\section{METHODS}

The density functional theory-based calculations were carried out using the QUANTUM ESPRESSO package [22]. I used the generalized gradient approximation (GGA) [23] of Perdew-Burke-Ernzerhof (PBE) [24]. The PBE functional was used even though it is known to underestimate the band gap [25,26]. This band-gap problem can be resolved by using a computationally expensive hybrid functional such as HSE06 $[27,28]$, which includes a fixed percentage of Hartree-Fock exchange in the xc functional. However, in this work the emphasis is on (i) identifying the deep defect states through their spatial localization, (ii) understanding how the nature of the optically active defect states and the involvement of boron atoms play key roles in the response of different defects to the strain, and hence (iii) explaining the trends and changes in the emission frequencies (instead of absolute numbers) as a function of applied strain. As I am mostly interested in aspects of the electronic structure properties of the defects, which are expected to remain the same for the two functionals, I used PBE rather than the prohibitively expensive HSE06 approximation.

Defects were created in a $6 \times 6 \times 1$ (72-atom) supercell of hBN. The Brillouin zone was sampled with a grid of $\Gamma$ centered, $4 \times 4 \times 1 k$ points according to the Monkhorst-Pack method [29]. These calculations yielded the single-particle molecular orbitals (MOs) of different defect centers in the layered material. The ordering of the defect states in each of the deep defects was obtained from the calculated KohnSham eigenstates around the band gap. After determining the ground-state properties for the unstrained structures, I applied compressive and tensile strain to the system that ranged from $-5 \%$ to $+5 \%$ (unless the system ceased to behave like a
QE). The strain percent is defined as $\left(a-a_{0}\right) / a_{0}$, with $a_{0}$ being the equilibrium lattice constant. For each strain level, the defective structures are fully relaxed with residual forces smaller than $10^{-4}$ Ry / a.u.

In order to study the effect of strain on the excitedstate properties, I calculated the ZPL for each of the defects under different levels of strain using the constrainedoccupation DFT (CDFT) method. Within the CDFT method, the occupation of the defect states is constrained to mimic the photoluminescence process shown in Fig. 1(a). This is done by promoting the electron from the lower-energy defect state involved in the excitation to the higher-energy defect state, which was previously unoccupied. The structure is then allowed to relax with this new electronic configuration. The total energy differences between different electronic and ionic configurations are then mapped onto the Franck-Condon picture, giving optical transition energies [30]. Within the Franck-Condon picture, it is assumed that the electronic transitions upon absorption or emission of a photon happen much faster as compared to the rearrangement of the nuclei. This is illustrated in Fig. 1(b), wherein the vertical transition $\mathrm{A} \rightarrow \mathrm{B}$ corresponds to excitation (vertical absorption), $\mathrm{C} \leftrightarrow$ A corresponds to the $\mathrm{ZPL}$, and $\mathrm{C} \rightarrow \mathrm{D}$ corresponds to the vertical electronic transition with an emission of a photon.

\section{RESULTS}

Out of all possible spin-active defects [31], I have selected three intrinsic defects of $\mathrm{hBN}$ : a neutral nitrogen vacancy $\left(V_{\mathrm{N}}^{0}\right)$, a negatively charged boron vacancy $\left(V_{\mathrm{B}}^{-1}\right)$, and a neutral antisite complex comprised of a nitrogen vacancy next to a nitrogen substitutional $\left(V_{\mathrm{N}} N_{\mathrm{B}}^{0}\right)$. The purpose of choosing these 
TABLE I. Properties of deep defects in $\mathrm{hBN}$ for strain-free structures: spin of the defect, spin polarization energies, $\mathrm{E}_{\mathrm{Pol} \text {. (i.e., }}$ difference in total energies of spin-unpolarized and spin-polarized calculations), and identity of the optically active majority-spin states and minority-spin states that are involved in the lowest-energy spinpreserving excitations.

\begin{tabular}{lccl}
\hline \hline System & Spin & $\begin{array}{c}E_{\text {Pol. }} \\
(\mathrm{meV})\end{array}$ & Optical Excitation \\
\hline$V_{\mathrm{N}}$ & $1 / 2$ & 143.03 & Majority Spin \\
$V_{\mathrm{B}}{ }^{-1}$ & 1 & 850.41 & Minority Spin \\
$V_{\mathrm{N}} N_{\mathrm{B}}$ & $1 / 2$ & 227.80 & Minority Spin \\
\hline \hline
\end{tabular}

particular defects is to demonstrate proof of principle. The relatively large formation energies [32] of two of the defects $\left(V_{\mathrm{N}}^{0}\right.$ and $V_{\mathrm{N}} N_{\mathrm{B}}^{0}$ ) would likely preclude them from existing in large concentrations naturally. The possibility of observing them increases markedly, however, in systems where the defects are formed by irradiation, a common technique for forming defects in experiments [7]. Through these defects, I demonstrate that the strain not only tunes excitation energies, but it can also be used to characterize defects through their response to the applied strain, which along with other pieces of information, such as symmetry and/or spin, can be used to identify the defects.

The two simple point defects studied in this work, $V_{\mathrm{N}}^{0}$ and $V_{\mathrm{B}}^{-1}$, have the same symmetry $\left(D_{3 h}\right)$, although they have different spins. On the other hand, $V_{\mathrm{N}}^{0}$ and $V_{\mathrm{N}} N_{\mathrm{B}}^{0}$ have the same spin, although they have different symmetries. Each of these defects chosen in this study has partially filled defect states derived from the dangling $s p^{2}$-hybridized bonds. The spatial localization of the defect states [see Fig. 1(c)] results in a large exchange interaction and hence, in spin-polarized structures with more electrons in one spin channel (majority spin) than the other (minority-spin channel) [33,34]. For the strain-free structures, the spin polarization energies, $E_{\mathrm{Pol}}$. (= difference in total energies of spin-unpolarized and spinpolarized calculations), are summarized in Table I. As the calculated $E_{\text {Pol. }}$ values are much greater than the thermal energy at room temperature $\left(k_{B} T \approx 25 \mathrm{meV}\right)$, the spins in these systems are expected to survive well beyond room temperature, making them promising qubit candidates. In these defects, there can be several possible spin-preserving optical excitations between the defect states. In Table I, I identify the spin channel involved in the lowest-energy optical excitations. In the remainder of the work, we concentrate on the effect of strain on these optically active defect states involved in the lowest-energy excitations.

\section{A. Neutral nitrogen vacancy}

$V_{\mathrm{N}}^{0}$ is a spin-1/2 defect with a $D_{3 h}$ point-group symmetry. At $0 \%$ strain the undercoordinated boron atoms surrounding the defect maximize their interactions by moving inwards towards the defect (boron-boron distance $d_{\mathrm{BB}}=2.29 \AA$, as compared to $2.51 \AA$ in an ideal crystal). Figure 2(a) shows the optically active majority-spin states for $V_{\mathrm{N}}^{0}$ corresponding to (a)

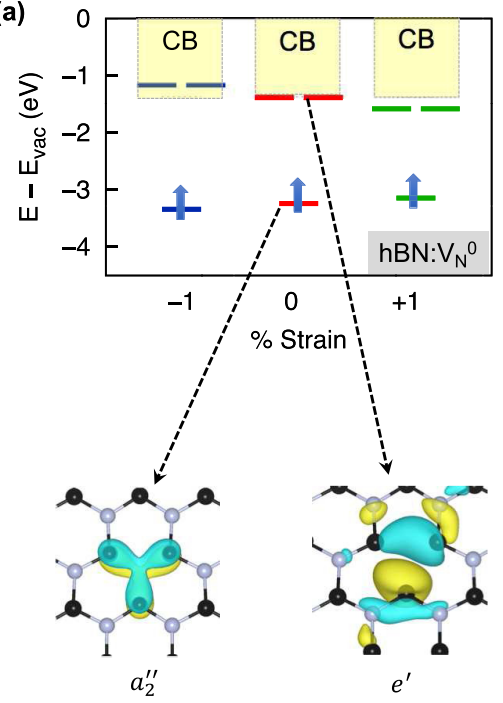

(b)

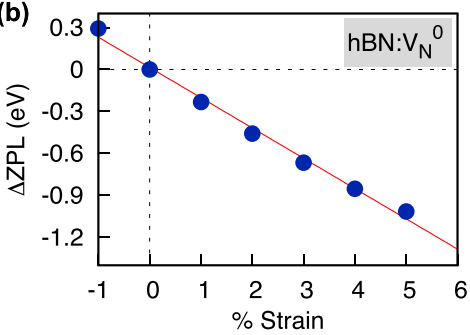

(c)

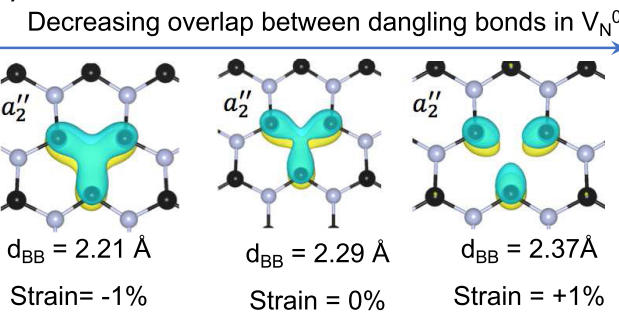

(d)

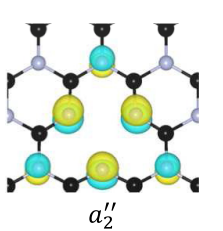
$\mathrm{hBN}: \mathrm{V}_{\mathrm{B}}^{-1}$

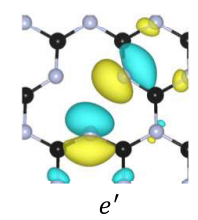

(e)

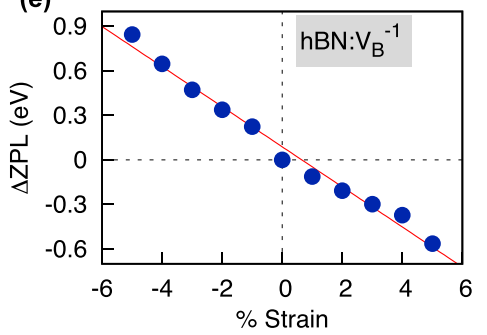

FIG. 2. Strain-tuned excitation energies of simple point defects. (a) Single-particle energy levels of the defect states involved in the optical excitation in $\mathrm{V}_{\mathrm{N}}^{0}$ as a function of strain with the vacuum energy used as the reference energy. Optical excitation corresponds to promotion of electrons from a filled $a_{2}^{\prime \prime}$ state (singlet) to the empty $e^{\prime}$ states (doublet) in the spin-up channel. CB here stands for conduction band. Also shown are the charge density plots for the singlet and one of the doublets. (b) Change in the ZPL of $V_{\mathrm{N}}^{0}$ as a function of strain (ZPL at $0 \%$ strain used as reference), with the red line being the best fit. (c) Decreasing overlap between the dangling bonds with the expansion of the lattice (only $a_{2}^{\prime \prime}$ state shown) increases (decreases) the energy of bondinglike (antibonding) orbitals. Here, $d_{\mathrm{BB}}$ is the distance between boron atoms around the defect. (d) Optically active defect states involved in the excitation in $\mathrm{V}_{\mathrm{B}}^{-1}$. Excitation takes the electron from the filled $a_{2}^{\prime \prime}$ state (singlet) to the empty $e^{\prime}$ (doublet) states (only one of the $e^{\prime}$ states shown). (e) Changes in the ZPL of $V_{\mathrm{B}}^{-1}$ as a function of strain, with the red line being the best linear fit. 
the filled $a_{2}^{\prime \prime}$ state (singlet) and the empty $e^{\prime}$ state (doublet) for three different strains. The level ordering obtained for $V_{\mathrm{N}}^{0}$ within the PBE approximation agrees with the ordering obtained via a more rigorous approach employing the stateof-the-art GW approximation [35]. Charge density plots of the optically active states in Fig. 2(a) show the bonding character of the $a_{2}^{\prime \prime}$ state and the antibonding character of the $e^{\prime}$ state. Dilation (contraction) of the lattice results in narrowing (widening) of the gap between the $a_{2}^{\prime \prime}$ state and the empty $e^{\prime}$ state. Beyond $1 \%$ compressive strain, the empty $e^{\prime}$-defect state hybridizes with the bulk states. In experiments, this will appear as a loss of signal from the quantum emitter. These strain-induced changes in separation of optically active levels result in corresponding changes in the ZPL. Figure 2(b) shows that the ZPL is linearly dependent on the applied strain, changing at the rate of $\sim 0.216 \mathrm{eV} / \%$ strain. This can be understood within molecular orbital theory. As seen in the isosurface plots for $a_{2}^{\prime \prime}$ states in Fig. 2(c), lattice expansion increases the distance between boron atoms surrounding the defect. This decreases the overlap between their dangling bonds that make up the molecular orbitals. In turn, this reduction in overlap increases the energy of $a_{2}^{\prime \prime}$, which is mostly bonding in character, while the energy of the $e^{\prime}$ states, which are antibonding in character, simultaneously decreases. As a result, the energy difference between the states involved in the excitation is reduced, lowering the ZPL values when tensile strain is applied.

\section{B. Negatively charged boron vacancy}

$V_{\mathrm{B}}^{-1}$ is a spin-1 defect with a $D_{3 h}$ point-group symmetry. The smallest-energy excitation takes place between a filled $a_{2}^{\prime \prime}$ state (singlet) and an empty $e^{\prime}$ state (doublet) in the minority-spin channel [Fig. 2(d)]. The dependence of the ZPL on strain shows monotonic behavior, as seen in Fig. 2(e). The energy of quantum emission from the defect decreases (increases) upon lattice dilation (contraction) at the rate of $\sim 0.138 \mathrm{eV} / \%$ strain. The filled $a_{2}^{\prime \prime}$ state is derived from the dangling $\pi$ orbitals associated with the nearest-neighbor $(\mathrm{NN})$ nitrogen atoms and the next-to-nearest neighbor (NNN) nitrogen atoms surrounding the defect [Fig. 2(d)]. The $a_{2}^{\prime \prime}$ state is partially bonding (NN nitrogens) and partially antibonding (between $\mathrm{NN}$ and $\mathrm{NNN}$ nitrogens) in character. As a result, lattice compression and dilation have a smaller effect on the energy of this level. On the other hand, the empty $e^{\prime}$ state is mostly derived from the dangling $\sigma$ orbitals associated with NN nitrogen atoms and is chiefly antibonding in character. As a consequence, lattice dilation (compression) lowers (increases) its energy. This also explains the different rate at which the ZPL of $V_{\mathrm{B}}^{-1}$ and $V_{\mathrm{N}}^{0}$ are changed upon application of strain. For $V_{\mathrm{N}}^{0}$, which shows an enhanced response to the strain, the optically active states have opposite character (bonding vs antibonding), and both states simultaneously change their energies in the opposite sense upon application of strain. There is some contribution to the $e^{\prime}$ states of $V_{\mathrm{B}}^{-1}$ coming from the boron atoms as well [Fig. 2(d)], and this small bonding-character introduced by these boron atoms plays a role, as will be discussed later.

\section{Neutral antisite complex}

$V_{\mathrm{N}} N_{\mathrm{B}}^{0}$ is a spin- $1 / 2$ defect with a $C_{2 V}$ point-group symmetry. The antisite complex is considered to be one of the possible defects that emits in the visible range $[6,9,21,32]$. In the ground-state equilibrium geometry (GEG), the two boron atoms surrounding $V_{\mathrm{N}} N_{\mathrm{B}}^{0}$ form a weak covalent bond, with $d_{\mathrm{BB}}=1.94 \AA$ for $0 \%$ strain. The lowest-energy excitation corresponds to the promotion of an electron from a filled singlet $\left(a_{1}\right)$ state to an empty singlet $\left(b_{2}\right)$ state in the minorityspin channel. Figure 3(a) gives the defect levels involved in the excitation for different strains, along with their charge density plots. The $a_{1}$ state, which shows antibonding character between the dominant contributing centers $\left(\mathrm{N}_{\mathrm{B}}\right.$ and the two bonded borons), is stabilized (destabilized) upon dilatation (contraction). On the other hand, the $b_{2}$ state, for which the charge density is mostly localized on the antisite nitrogen atom, is minimally affected by strain. Figure 3(b) shows changes in the ZPL as a function of strain. The ZPL has a nonmonotonic behavior, which is unlike the trends shown by $V_{\mathrm{B}}^{-1}$ and $V_{\mathrm{N}}^{0}$, distinguishing this defect from other intrinsic point defects. This is also different from what one might expect from the increasing energy differences in the $a_{1}$ and the $b_{2}$ states with lattice expansion [see Fig. 3(a)]. Interestingly, the theoretically predicted nonmonotonic behavior of the ZPL agrees with the results obtained experimentally by Grosso et al. [9]. In Ref. [9], the authors suspected that the antisite complex is their defect and studied the strain dependence of its ZPL, finding nonmonotonic variation in emission frequencies as a function of strain. In order to quantitatively compare theoretical results presented here with the experimental results, one needs to account for the large thickness of hBN samples ( $\sim 150$ layers) used in Ref. [9] and the initial conditions of the $\mathrm{hBN}$ flakes. These samples likely did not start out strain-free but had different built-in strain levels due to several reasons, such as wrinkles or cracks in the samples. For three of these quantum emitters (all assumed to be antisite complexes), Grosso et al. found that the rate of ZPL shift varies from -3.1 to $6.0 \mathrm{meV} / \%$ strain [9]. Assuming that only about $98 \%$ of strain is transferred between any two consecutive layers [36], a much smaller strain was actually transmitted to the upper layers containing the emitters as compared to the strain applied to the bottom layer of their hBN flakes via a flexible substrate. This effective strain, $S_{\text {eff }}$, transmitted to the $N$ th layer, can be estimated using [9] $S_{\text {eff }}=t^{N} \times S_{\text {app }} \approx$ $0.05 S_{\text {app }}$. Here, $t \sim 98 \%$ is the strain transferred between two consecutive layers, $N \sim 150$ is the number of layers, and $S_{\text {app }}$ is the strain applied to the substrate. Thus, the rates of ZPL shifts measured for different antisite complexes in Ref. [9] were underestimated by more than an order of magnitude. If one takes into account two factors-the incomplete transmittal of strain between layers and the pre-existing strain within the hBN samples-one finds that the theoretical results presented here are consistent with the experimental results in Ref. [9]. For example, from the best fit curve (quadratic) for this defect [plotted in Fig. 3(b)], one obtains a rate of ZPL shift (slope) to be $\sim-31.5 \mathrm{meV} / \%$ at about $+2.35 \%$ strain value, while a rate of $\sim+60.0 \mathrm{meV} / \%$ is obtained at about $-0.5 \%$ strain. The agreement between my results presented here and the experimental work by Grosso et al. fur- 

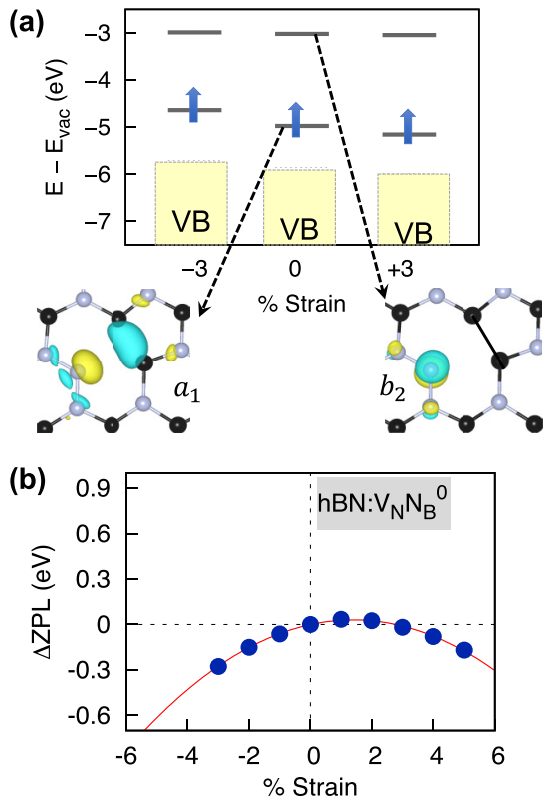
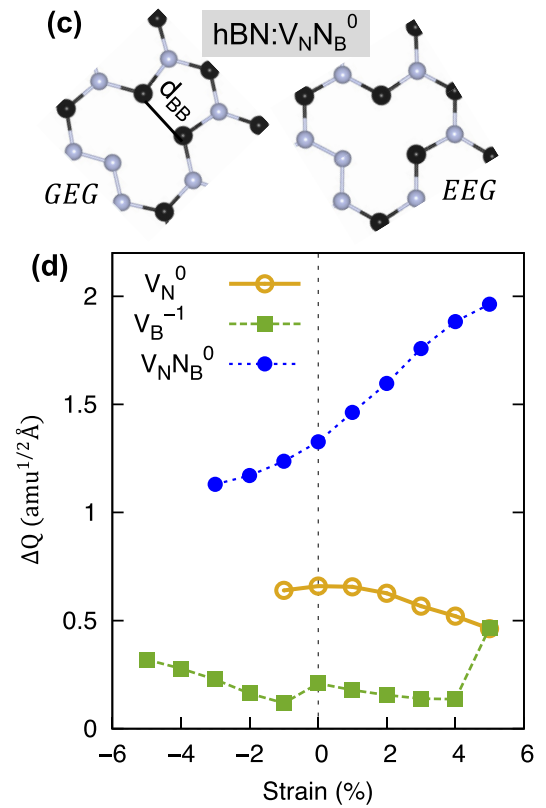

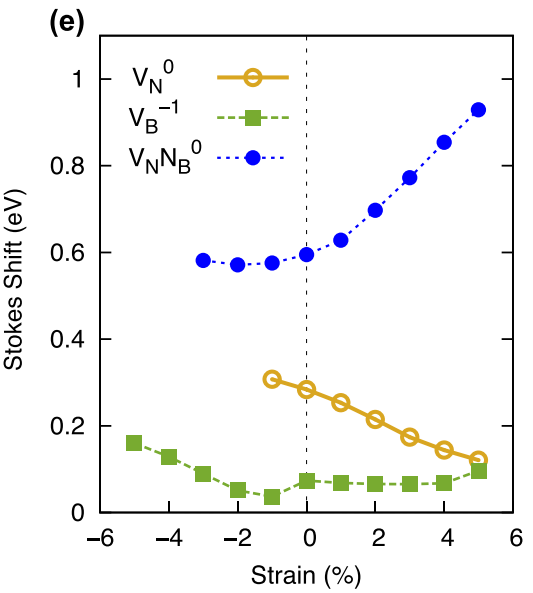

FIG. 3. Distinctive response to strain of the antisite complex. (a) Single-particle energy levels of the defect states involved in the optical excitation in $V_{\mathrm{N}} N_{\mathrm{B}}^{0}$ as a function of strain, with the vacuum energy used as the reference energy. Optical excitation corresponds to promotion of electrons from a filled $a_{1}$ state (singlet) to the empty $b_{2}$ state (singlet) in the minority-spin channel. VB here stands for valence band. (b) Change in the ZPL of $V_{\mathrm{N}} N_{\mathrm{B}}^{0}$ as a function of strain (with ZPL at $0 \%$ strain as reference), with the red line being the best fit, showing the nonmonotonic behavior of the ZPL. (c) The ground-state equilibrium geometry (GEG) and the excited-state equilibrium geometry (EEG) of $V_{\mathrm{N}} N_{\mathrm{B}}^{0}$, showing the change in distances $\left(d_{\mathrm{BB}}\right)$ between the weakly bonded boron atoms. (d) The change in geometry between ground and excited states is quantified by the change in generalized configuration coordinate $\Delta \mathrm{Q}$ and plotted as a function of strain. (e) Strain tuning of the Stokes shift (difference between excitation energy and the ZPL).

ther validates their assumption that their defect is an antisite complex.

\section{DISCUSSION}

The unexpected trend in the strain-tuned ZPL for $V_{\mathrm{N}} N_{\mathrm{B}}^{0}$ comes from the rich chemistry of the boron atoms that are involved in the defect. Changes in electronic configuration upon excitation dictate the changes in atomic structure in the excited state. In turn, the strain affects the extent of change in the atomic structures due to excitation. As seen in Fig. 3(c), the excited-state equilibrium geometry (EEG) for $V_{\mathrm{N}} N_{\mathrm{B}}^{0}$ shows a large change in boron-boron distance $\left(d_{\mathrm{BB}}=2.28 \mathrm{~A}\right)$. The difference in GEG and EEG can be quantified by calculating the change in the generalized configuration coordinate, $\Delta \mathrm{Q}$, given by the formula $\Delta \mathrm{Q}^{2}=\sum_{i \alpha} m_{\alpha}\left(R_{i \alpha}^{\mathrm{EEG}}-R_{i \alpha}^{\mathrm{GEG}}\right)^{2}$. Here, $m_{\alpha}$ is the atomic mass of the $\alpha$ th atom, and $\left(R_{i \alpha}^{\mathrm{EEG}}-R_{i \alpha}^{\mathrm{GEG}}\right)$ is its displacement in the $i$ th direction $(i=x, y, z)$. In the case of lattice contraction, the changes in the geometry of $V_{\mathrm{N}} N_{\mathrm{B}}^{0}$ are small due to the strengthening of the boron-boron bond, while lattice dilation leads to large changes in the geometry due to the weakening of the boron-boron bond [Fig. 3(d)]. This is different from the strain dependence of $\Delta Q$ for the two simple point defects. In the case of $V_{\mathrm{N}}^{0}$, the distance between neighboring boron atoms changes from $2.29 \AA$ in ground state to $2.47 \AA$ in the excited state for $0 \%$ strain. Since these atoms are not bonded, there is a much smaller change in $\Delta \mathrm{Q}$ with strain as compared to the antisite complex. This is also true of $V_{\mathrm{B}}^{-1}$, in which case an even smaller role is played by the interactions between the nonbonded boron atoms that are next-to-nearest neighboring atoms surrounding the defect. The small, but non-negligible contribution of the boron atoms to the $e^{\prime}$ (doublet) states involved in the excitation [Fig. 2(d)] is responsible for the small deviations from the linear fit for the strain-tuning of the ZPL [Fig. 2(e)] and is reflected in the strain dependence of $\Delta \mathrm{Q}$ for $V_{\mathrm{B}}^{-1}$. Figure 3(e) shows the strain-dependent Stokes shift (difference in the vertical excitation and the ZPL) for the three defects. The much larger change in the strain-tuned Stokes shift for $V_{\mathrm{N}} N_{\mathrm{B}}^{0}$ as compared to the two other defects further supports the above analysis. It also explains the unexpected decrease in the ZPL of $V_{\mathrm{N}} N_{\mathrm{B}}^{0}$ with lattice dilation [Fig. 3(b)]. This nonmonotonic behavior of the ZPL is due to the large increase in Stokes shift with increasing dilation.

\section{CONCLUSION}

In summary, I demonstrated that the response of the defects to strain depends on the detailed nature of the defect states involved in the optical excitations and the rich boron chemistry, which results in complex interactions between the boron atoms. Strain not only tunes emission frequencies but also provides a possible way of fingerprinting defects responsible for quantum emission. Identifying defects and better understanding their characteristic responses to external stimuli such as strain, in turn, will help to harness their potential in the fields of quantum information, quantum sensing, and quantum photonics. 


\section{ACKNOWLEDGMENTS}

P.D. thanks P. Zhang (University at Buffalo, Buffalo, NY) for discussions. This work is supported by NSF Grant No. DMR-1752840 and the STC Center for Integrated Quantum Materials under NSF Grant No. DMR1231319. P.D. acknowledges the computational support provided by the Extreme Science and Engineering Discovery Environment (XSEDE) under Project No. PHY180014, which is supported by National Science Foundation Grant No. ACI-1548562. For three-dimensional visualization of crystals and volumetric data, use of VESTA 3 software is acknowledged.
[1] A. Srivastava, M. Sidler, A. V. Allain, D. S. Lembke, A. Kis, and A. Imamoğlu, Optically active quantum dots in monolayer $\mathrm{WSe}_{2}$, Nat. Nanotechnol. 10, 491 (2015).

[2] Y.-M. He, G. Clark, J. R. Schaibley, Y. He, M.-C. Chen, Yu.-J. Wei, X. Ding, Q. Zhang, W. Yao, X. Xu, C.-Y. Lu, and J.-W. Pan, Single quantum emitters in monolayer semiconductors, Nat. Nanotechnol. 10, 497 (2015).

[3] C. Chakraborty, L. Kinnischtzke, K. M. Goodfellow, R. Beams, and A. N. Vamivakas, Voltage-controlled quantum light from an atomically thin semiconductor, Nat. Nanotechnol. 10, 507 (2015).

[4] M. Koperski, K. Nogajewski, A. Arora, V. Cherkez, P. Mallet, J.-Y. Veuillen, J. Marcus, P. Kossacki, and M. Potemski, Single photon emitters in exfoliated $\mathrm{WSe}_{2}$ structures, Nat. Nanotechnol. 10, 503 (2015).

[5] T. T. Tran, K. Bray, M. J. Ford, M. Toth, and I. Aharonovich, Quantum emission from hexagonal boron nitride monolayers, Nat. Nanotechnol. 11, 37 (2015).

[6] T. T. Tran, C. Elbadawi, D. Totonjian, C. J. Lobo, G. Grosso, H. Moon, D. R. Englund, M. J. Ford, I. Aharonovich, and M. Toth, Robust multicolor single photon emission from point defects in hexagonal boron nitride, ACS Nano 10, 7331 (2016).

[7] S. Choi, T. T. Tran, C. Elbadawi, C. Lobo, X. Wang, S. Juodkazis, G. Seniutinas, M. Toth, and I. Aharonovich, Engineering and localization of quantum emitters in large hexagonal boron nitride layers, ACS Appl. Mater. Interfaces 8, 29642 (2016).

[8] L. J. Martínez, T. Pelini, V. Waselowski, J. R. Maze, B. Gil, G. Cassabois, and V. Jacques, Efficient single photon emission from a high-purity hexagonal boron nitride crystal, Phys. Rev. B 94, 121405(R) (2016).

[9] G. Grosso, H. Moon, B. Lienhard, S. Ali, D. K. Efetov, M. M. Furchi, P. Jarillo-Herrero, M. J. Ford, I. Aharonovich, and D. Englund, Tunable and high purity room-temperature single photon emission from atomic defects in hexagonal boron nitride, Nat. Commun. 8, 705 (2017).

[10] C. Palacios-Berraquero, D. M. Kara, A. R.-P. Montblanch, M. Barbone, P. Latawiec, D. Yoon, A. K. Ott, M. Loncar, A. C. Ferrari, and M. Atatüre, Large-scale quantum-emitter arrays in atomically thin semiconductors, Nat. Commun. 8, 15093 (2017).

[11] A. L. Exarhos, D. A. Hopper, R. R. Grote, A. Alkauskas, and L. C. Bassett, Optical signatures of quantum emitters in suspended hexagonal boron nitride, ACS Nano 11, 3328 (2017).

[12] T. T. Tran, M. Kianinia, M. Nguyen, S. Kim, Z.-Q. Xu, A. Kubanek, M. Toth, and I. Aharonovich, Resonant excitation of quantum emitters in hexagonal boron nitride, ACS Photonics $\mathbf{5}$, 295 (2018)
[13] C. D. Cress, S. W. Schmucker, A. L. Friedman, P. Dev, J. C. Culbertson, J. W. Lyding, and J. T. Robinson, Nitrogen-doped graphene and twisted bilayer graphene via hyperthermal ion implantation with depth control, ACS Nano 10, 3714 (2016).

[14] C. R. Dean, A. F. Young, I. Meric, C. Lee, L. Wang, S Sorgenfrei, K. Watanabe, T. Taniguchi, P. Kim, K. L. Shepard, and J. Hone, Boron nitride substrates for high-quality graphene electronics, Nat. Nanotechnol. 5, 722 (2010).

[15] A. K. Geim and I. V. Grigorieva, Van der waals heterostructures, Nature (London) 499, 419 (2013).

[16] X. Cui, G.-H. Lee, Y. D. Kim, G. Arefe, P. Y. Huang, C.-H. Lee, D. A. Chenet, X. Zhang, L. Wang, F. Ye, F. Pizzocchero, B. S. Jessen, K. Watanabe, T. Taniguchi, D. A. Muller, T. Low, P. Kim, and J. Hone, Multi-terminal transport measurements of MoS2 using a van der Waals heterostructure device platform, Nat. Nanotechnol. 10, 534 (2015).

[17] Z. Shotan, H. Jayakumar, C. R. Considine, M. Mackoit, H. Fedder, J. Wrachtrup, A. Alkauskas, M. W. Doherty, V. M. Menon, and C. A. Meriles, Photoinduced modification of single-photon emitters in hexagonal boron nitride, ACS Photonics 3, 2490 (2016).

[18] N. R. Jungwirth, B. Calderon, Y. Ji, M. G. Spencer, M. E. Flatté, and G. D. Fuchs, Temperature dependence of wavelength selectable zero-phonon emission from single defects in hexagonal boron nitride, Nano Lett. 16, 6052 (2016).

[19] T. Vogl, M. W. Doherty, B. C. Buchler, Y. Lu, and P. K. Lam, Atomic localization of quantum emitters in multilayer hexagonal boron nitride, Nanoscale 11, 14362 (2019).

[20] T. Vogl, G. Campbell, B. C. Buchler, Y. Lu, and P. K. Lam, Fabrication and deterministic transfer of high-quality quantum emitters in hexagonal boron nitride, ACS Photonics 5, 2305 (2018).

[21] S. Lazić, A. Espinha, S. Pinilla Yanguas, C. Gibaja, F. Zamora, P. Ares, M. Chhowalla, W. S. Paz, J. J. P. Burgos, A. HernándezMínguez, P. V. Santos, and H. P. van der Meulen, Dynamically tuned non-classical light emission from atomic defects in hexagonal boron nitride, Commun. Phys. 2, 113 (2019).

[22] P. Giannozzi, S. Baroni, N. Bonini, M. Calandra, R. Car, C. Cavazzoni, D. Ceresoli, G. L. Chiarotti, M. Cococcioni, I. Dabo, A. Dal Corso, S. de Gironcoli, S. Fabris, G. Fratesi, R. Gebauer, U. Gerstmann, C. Gougoussis, A. Kokalj, M. Lazzeri, L. Martin-Samos et al., Quantum espresso: A modular and open-source software project for quantum simulations of materials, J. Phys.: Condens. Matter 21, 395502 (2009).

[23] J. P. Perdew and Y. Wang, Accurate and simple density functional for the electronic exchange energy: Generalized gradient approximation, Phys. Rev. B 33, 8800 (1986).

[24] J. P. Perdew, K. Burke, and M. Ernzerhof, Generalized Gradient Approximation Made Simple, Phys. Rev. Lett. 77, 3865 (1996). 
[25] A. J. Cohen, P. Mori-Sánchez, and W. Yang, Insights into current limitations of density functional theory, Science 321, 792 (2008).

[26] B. Arnaud, S. Lebègue, P. Rabiller, and M. Alouani, Huge Excitonic Effects in Layered Hexagonal Boron Nitride, Phys. Rev. Lett. 96, 026402 (2006).

[27] J. Heyd, G. E. Scuseria, and M. Ernzerhof, Hybrid functionals based on a screened coulomb potential, J. Chem. Phys. 118, 8207 (2003).

[28] J. Heyd, G. E. Scuseria, and M. Ernzerhof, Erratum: Hybrid functionals based on a screened Coulomb potential [J. Chem. Phys. 118, 8207 (2003)], J. Chem. Phys. 124, 219906 (2006).

[29] H. J. Monkhorst and J. D. Pack, Special points for Brillouinzone integrations, Phys. Rev. B 13, 5188 (1976).

[30] A. Gali, E. Janzen, P. Deak, G. Kresse, and E. Kaxiras, Theory of Spin-Conserving Excitation of the N-V Center in Diamond, Phys. Rev. Lett. 103, 186404 (2009).

[31] S. A. Tawfik, S. Ali, M. Fronzi, M. Kianinia, T. T. Tran, C. Stampfl, I. Aharonovich, M. Toth, and M. J. Ford,
First-principles investigation of quantum emission from $\mathrm{hBN}$ defects, Nanoscale 9, 13575 (2017).

[32] L. Weston, D. Wickramaratne, M. Mackoit, A. Alkauskas, and C. G. Van de Walle, Native point defects and impurities in hexagonal boron nitride, Phys. Rev. B 97, 214104 (2018).

[33] P. Dev, Y. Xue, and P. Zhang, Defect-Induced Intrinsic Magnetism in Wide-Gap III Nitrides, Phys. Rev. Lett. 100, 117204 (2008).

[34] P. Dev and P. Zhang, Unconventional magnetism in semiconductors: Role of localized acceptor states, Phys. Rev. B 81, 085207 (2010).

[35] C. Attaccalite, M. Bockstedte, A. Marini, A. Rubio, and L. Wirtz, Coupling of excitons and defect states in boron-nitride nanostructures, Phys. Rev. B 83, 144115 (2011).

[36] H. Kumar, L. Dong, and V. B. Shenoy, Limits of coherency and strain transfer in flexible 2D van der Waals heterostructures: Formation of strain solitons and interlayer debonding, Sci. Rep. 6, 21516 (2016). 